\title{
Taxonomic Studies of Two Common Poisonous Plants
}

\author{
Kranti Rai ${ }^{1}$, Dr. Ela Tiwari ${ }^{2}$ \\ Research scholar \& Prof. Deptt. Of Botany \\ ${ }^{1,2}$ (Department Of Botany/ Government Autonomous P.G. College of Excellence/University Of Sagar MP/India)
}

\begin{abstract}
Plants which are harmful to human to human or animal body are known poisonous. The plants are poisonously effective on contact, ingestion or by absorption or inhalation. These plants are used in the form of potential killers. Tropical dry deciduous forests are the home of many of these plants. The present study includes the taxonomy, toxic and medicinal properties of some poisonous plants of this region. The poisonous plants have toxic properties because they contain many alkaloids, ricins, atropines, scopolamine, brucin, lactins, etc. poisonous plants have many properties like narcotics, delirients, irritants, depressants, astenics, purgatives etc. many of these contents are used for the preparation of medicines as well. Some common poisonous plants are Oleander (Nerium oledander), Bacain (Melia azadirach), Arund (Ricinus communis), Dhatura (Datura metel, D.alba, D. Stramonium) Lantana Camera, Madar (Calotropis procera), Abrus precatorius etc.
\end{abstract}

Keywords: Second $=n d$, edition $=e d s$, figure $=$ fig, Length $=l$, breadth $=b$

\section{Introduction}

In the form of vital source, plants are essential part of our life. Many plants contain a large number of biologically active chemicals which are extremely useful for treating various human and animal diseases. While some plants, trees, herbs are deadly poisons and cause serious illness of men and animals. Plant toxicity is caused by toxic constituents i.e. Alkaloids, glycosides, saponins, tannins, organic acids. Which effect by contact, eaten, absorption or inhalation; they cause painful skin irritations, internal poisoning and undesirable affects upon men and animals. The taxonomic, morphological and phytochemical characters of poisonous plants have been described by Smith (1923), and Caius (2003). Faust and Jones (1973) found that trichomes can be diagnostic characteristics in Vernonia for their identification. General structures of trichomes have been discussed by Ramayya et.al. (1962), Sahu (1982, 83, 84, 85), Tiwari (1982). They indicated that the morphological characters of trichomes provide data for identification of plants at generic, specific or intraspecific level. Stomata in some Cucurbitaceae have reported by Inamdar etal. (1976). On the basis of arrangement of the epidermal cell neighboring the guard cell, more than 25 main types of stomata in dicots have been recognized (Metcalfe \& Chalk, 1979).Therefore the present work has been undertaken which deals with the structure, organographic distribution and phylogenic considerations of stomata and trichomes of poisonous plants.

\section{Materials and methods}

In the central India, hills of Sagar district are made from Vindhya and Basalt rocks. Forests types of Sagar district are tropical dry deciduous forest (Champian and Seth 1968).

The following materials of the poisonous plants were taken for study:-

Thevetia neriifolia

Thevetia peruviana

The taxonomic, morphological, and phytochemical study of poisonous plants has been done.

Trichomes and stomata structure had studied by the methods of Bobous and Beakbane (1971) under microscope and camera lucida diagrams were prepared.

The Stomata index was calculated according to the following formula:

(1) Stomata index\% (SI) $=\quad$ stomata density $* 100$

(Stomata density + epidermal cell density)

(Salisbury, 1927).

Where: [Area of grid $=5 * 5=25$ square micron (where objective lens is $10 \mathrm{x}$ and eye lens is $15 \mathrm{x}$ )

Area of grid $=1.25 * 1.25=1.56$ square micron (where objective lens is $40 \mathrm{x}$ and eye lens is $15 \mathrm{x}$ ).]

(2) Stomata density =

stomata frequency

$10 *$ area of grid square micron

(3) Stomata frequency $=$ number of stomata per unit area

From the epidermal cell count data, the trichome index was calculated according to the following formula: 
Where:-[Area of grid $=5 * 5=25$ square micron (where objective lens is $10 \mathrm{x}$ and eye lens is $15 \mathrm{x}$ )

Area of grid $=1.25^{*} 1.25=1.56$ square micron (where objective lens is $40 \mathrm{x}$ and eye lens is $15 \mathrm{x}$ ).]

(5) Trichome density $=$ trichome frequency

(6) Trichome frequency $=$ number of trichome per unit area

\subsection{Trichome}

2.1.1. Foot

The proximal foot is lying in the epidermis. It is recognized into two kinds viz.

Simple foot: may be as many cells as the number of the immediately overlaying part of the trichome.

Compound foot: may be cells which are more in number than the cell rows of the immediately overlaying part of the trichome.

2.1.2. Body

Distal part of the trichome is lying above the foot. It is recognized into two kinds viz.

Undifferentiated body: It is entire body.

Differentiated body: It is differentiated into two or more parts. The proximal body is stalked and distal body is head.

\subsection{Stomata}

A stoma is a pore, found in the leaf and stem epidermis. The pore is formed by a pair of parenchyma cells called guard cells that are response to given environmental conditions.

Morphologically, four main types of stomata have been distinguished in the dicotyledons on the basis of the arrangement of the epidermal cells neighboring the guard cells (Metcalfe and Chalk 1950).

\subsection{Thevetia peruviana}

\section{Observation}

\subsubsection{Scientific classification}

Kingdom: Plantae

Division: Tracheophyta

Class: Magnoliopsida

Order: Gentianales

Family: Apocynaceae

Genus: Thevetia

Species: Peruviana

\subsubsection{Morphological characters}

It is a large yellow flowered, evergreen shrub or small tree. The stem/trunk is green turning silver/gray as it ages. The leaves are willow: like, linear: lanceolate, acute or sub obtuse, sessile, dark green, glossy. The flowers are regular fragrant, bloom from summer to fall. The long funnel shaped, yellow flowers are in terminal cluster. The fruit is hard capsule and deep red black in color.

\subsubsection{Trichome characters}

Trichome plate A, fig 1, 3, 4,9,10. Multicelled flagellate glandular hair. Description: foot compound, body multicelled, differentiated into stalk and head, flagellate; contents translucent; walls thin, smooth and straight. Distribution: on style, carpel, pedicel, corolla, fruitwall, and petal. Trichome plate A, fig 5, 8, 6 . Unicelled flagellate glandular hair.

Description: foot compound, body unicelled, undifferentiated, flagellate, pointed apex; contents translucent; walls thin, smooth and straight.

Distribution: on stigma, anther, and fruitwall.

Trichome plate A, fig 2, 7. Papillate glandular hair.

Description: foot compound, body papillose, oblique, entire, spathulate apex; contents translucent; walls thin, smooth and straight.

Distribution: on stigma, anther, carpel and sepal.

3.1.4. Stomata characters

There are following stomata that distributed on different parts of thevetia pereviana.

Anomocytic, anomotetracytic stomata.

Distribution: leaf

anomocytic stomata.

Distribution: stem, pedicel, calyx.

Brachyparacytic stomata. 
Distribution: corolla.

anomotetracytic stomata.

Distribution: fruit wall

3.1.5. Constituents

It contains cardiac glycosides, therein a and $b$, thevetoxin, peruvoside, ruvoside and nerifolin.

3.1.6. Medicinal properties

It is applied medicinally for treatment of cardiac insufficiency.

3.1.7. Toxic effects

It may develop digitalis poisoning with gastrointestinal and cardiac symptoms, drowsiness, mydriasis, coma and convulsions.

3.2. Thevetia neriifolia

3.2.1. Scientific classification

Kingdom: Plantae

Division: Tracheophyta

Class: Magnoliopsida

Order: Gentianales

Family: Apocynaceae

Genus: Thevetia

Species: Neriifolia

3.2.2. Morphological characters

It is a bush, shrub or small tree. The leaves are glossy dark green, linear lanceolate. The flowers are long funnel shaped, fragrant, and orange in color.

3.2.3. Trichome characters

Trichome plate B, fig 1, 3; trichome plate c, fig 5,8,10. Unicelled flagellate glandular hair.

Description: foot compound, body flagellate, oblique, entire, rounded tip; contents translucent; walls thin, smooth and straight.

Distribution: on stem sepal, leaf, fruitwall, filament, and pedicel.

Trichome plate B, fig 2. Two celled flagellate glandular hair.

Description: foot compound, body entire, two celled, flagellate; contents translucent; walls thin, smooth and straight.

Distribution: on petal, leaf.

Trichome plate C, fig1. Multicelled branched glandular hair.

Description: foot compound, body uniseriate, differentiated into stalk and head, obliquely or vertically

subdivided in many cells, truncated apex; contents translucent; walls thin, smooth and straight.

Distribution: on anther lobe.

Trichome plate C, fig2. Two celled cylindrical glandular hair:-

Description: foot compound, body uniseriate, differentiated into stalk and head, head club shaped rounded apex; contents translucent; walls thin, smooth and straight.

Distribution: on pedicel.

Trichome plate C, fig 3, 4, 6, 7. Unicelled papillate glandular hair:-

Description: foot compound, body unicelled, papillose, rounded tip; contents translucent; walls thin, smooth and straight.

Distribution: on stigma, style, and petal.

Trichome plate C, fig9. Uniseriate flagellate glandular hair.

Description: foot compound, body uniseriate, entire; contents translucent; walls thin, smooth and straight.

Distribution: on carpel.

3.2.4. Stomata characters

There are following stomata that distributed on different parts of thevetia neriifolia.

Anomocytic, anomotetracytic, brachyparacytic, anisocytic stomata:

Distribution: leaf

Anomocytic stomata.

Distribution: stem, pedicel

Anomocytic, brachyparacytic stomata

Distribution: calyx

Anomocytic stomata.

Distribution: corolla

Anomotetracytic, anomocytic, anisocytic, brachyparacytic stomata

Distribution: fruit wall

3.2.5. Constituents

It contains glycosides of oleic acid, therein, thevetoxin. 
3.2.6. Medicinal properties

It is used as insecticides, arachnicides. It is used as in medicines for febrifuges menstrual cycle. Tincture of the bark is also emetic and purgative.

3.2.7. Toxic properties

It is cardio toxic acting like digitalis.

\section{Table and graph}

4.1. There are presented number of stomata of studied plants in table 1

\begin{tabular}{|c|c|c|c|c|c|c|c|c|c|c|}
\hline $\begin{array}{l}\text { Name } \\
\text { of } \\
\text { plant }\end{array}$ & $\begin{array}{l}\text { Nam } \\
\mathrm{e} \text { of } \\
\text { plant } \\
\text { parts }\end{array}$ & $\begin{array}{l}\text { type of } \\
\text { stomata }\end{array}$ & $\begin{array}{l}\mathrm{Nu} \\
\mathrm{mb} \\
\mathrm{er} \\
\text { of } \\
\text { sto } \\
\mathrm{ma} \\
\text { ta }\end{array}$ & $\begin{array}{l}\text { Sto } \\
\text { mat } \\
\text { al } \\
\text { fre } \\
\text { que } \\
\text { ncy }\end{array}$ & $\begin{array}{l}\text { Sto } \\
\text { mat } \\
\text { al } \\
\text { den } \\
\text { sity }\end{array}$ & $\begin{array}{l}\text { Type of } \\
\text { epiderma } \\
1 \text { cell }\end{array}$ & $\begin{array}{l}\text { Num } \\
\text { ber } \\
\text { of } \\
\text { epid } \\
\text { erma } \\
1 \text { cell }\end{array}$ & $\begin{array}{l}\text { Fre } \\
\text { que } \\
\text { ncy } \\
\text { of } \\
\text { epi } \\
\text { der } \\
\text { mal } \\
\text { cell }\end{array}$ & $\begin{array}{l}\text { Den } \\
\text { sity } \\
\text { of } \\
\text { epid } \\
\text { erma } \\
1 \text { cell }\end{array}$ & $\begin{array}{l}\text { Sto } \\
\text { mat } \\
\text { al } \\
\text { inde } \\
x\end{array}$ \\
\hline $\begin{array}{l}\text { Theveti } \\
\text { a } \\
\text { peruvi } \\
\text { ana }\end{array}$ & $\begin{array}{l}\text { Upp } \\
\text { er } \\
\text { layer } \\
\text { of } \\
\text { leaf }\end{array}$ & $\begin{array}{l}\text { Anomoc } \\
\text { ytic, } \\
\text { anomotet } \\
\text { racytic }\end{array}$ & $\begin{array}{l}1 \\
\text { or } \\
\text { rar } \\
\text { e }\end{array}$ & .32 & .02 & $\begin{array}{l}\text { Rectangu } \\
\text { lar, } \\
\text { pentagon } \\
\text { al, } \\
\text { hexagon } \\
\text { al }\end{array}$ & 110 & $\begin{array}{l}70 . \\
51\end{array}$ & 4.52 & 0.44 \\
\hline $\begin{array}{l}\text { Thevetia } \\
\text { peruvian } \\
a\end{array}$ & $\begin{array}{l}\text { Low } \\
\text { er } \\
\text { layer } \\
\text { of } \\
\text { leaf }\end{array}$ & $\begin{array}{l}\text { Anomoc } \\
\text { ytic, } \\
\text { anomotet } \\
\text { racytic }\end{array}$ & $\begin{array}{l}11 \\
\text { or } \\
12\end{array}$ & $\begin{array}{c}7.3 \\
7\end{array}$ & .47 & “ & 120 & $\begin{array}{l}76 . \\
92\end{array}$ & 4.93 & 8.70 \\
\hline $\begin{array}{l}\text { Thevetia } \\
\text { peruvian } \\
\text { a }\end{array}$ & Stem & $\begin{array}{l}\text { Anomoc } \\
\text { ytic }\end{array}$ & $\begin{array}{l}1 \\
\text { or } \\
\text { rar } \\
\text { e } \\
\end{array}$ & .32 & .02 & $\begin{array}{l}\text { rectangul } \\
\text { ar }\end{array}$ & 160 & $\begin{array}{l}102 \\
.56\end{array}$ & 6.57 & 0.30 \\
\hline $\begin{array}{l}\text { Thevetia } \\
\text { peruvian } \\
\text { a }\end{array}$ & $\begin{array}{l}\text { Pedi } \\
\text { cel }\end{array}$ & $\begin{array}{l}\text { Anomoc } \\
\text { ytic }\end{array}$ & $\begin{array}{l}1 \\
\text { or } \\
\text { rar } \\
\text { e }\end{array}$ & .32 & .02 & " & 160 & $\begin{array}{l}102 \\
.56\end{array}$ & 6.57 & 0.30 \\
\hline $\begin{array}{l}\text { Thevetia } \\
\text { peruvian } \\
a\end{array}$ & $\begin{array}{l}\text { Caly } \\
\mathrm{x}\end{array}$ & $\begin{array}{l}\text { anomocy } \\
\text { tic }\end{array}$ & $\begin{array}{l}11 \\
\text { or } \\
12\end{array}$ & $\begin{array}{c}7.3 \\
7\end{array}$ & .47 & $\begin{array}{l}\text { Rectangu } \\
\text { lar, } \\
\text { pentagon } \\
\text { al, } \\
\text { hexagon } \\
\text { al, } \\
\text { polygona } \\
1\end{array}$ & 125 & $\begin{array}{l}80 . \\
13\end{array}$ & 5.14 & 8.38 \\
\hline $\begin{array}{l}\text { Thevetia } \\
\text { peruvian } \\
a\end{array}$ & $\begin{array}{l}\text { Coro } \\
\text { lla }\end{array}$ & $\begin{array}{l}\text { Brachyp } \\
\text { aracytic }\end{array}$ & 1 & $\begin{array}{c}0.6 \\
4\end{array}$ & $\begin{array}{c}0.0 \\
4\end{array}$ & $\begin{array}{l}\text { Rectangu } \\
\text { lar, } \\
\text { hexagon } \\
\text { al }\end{array}$ & 120 & $\begin{array}{l}76 . \\
92\end{array}$ & 4.93 & 0.80 \\
\hline $\begin{array}{l}\text { Thevetia } \\
\text { peruvian } \\
a\end{array}$ & $\begin{array}{l}\text { Gyn } \\
\text { ocei } \\
\text { um } \\
\end{array}$ & & & & & oval & 325 & $\begin{array}{l}208 \\
.33\end{array}$ & $\begin{array}{c}13.3 \\
5\end{array}$ & \\
\hline $\begin{array}{l}\text { Thevetia } \\
\text { peruvian } \\
a\end{array}$ & $\begin{array}{l}\text { Andr } \\
\text { ocei } \\
\text { um }\end{array}$ & & & & & $\begin{array}{l}\text { Oval and } \\
\text { rectangul } \\
\text { ar }\end{array}$ & 200 & $\begin{array}{l}128 \\
.21\end{array}$ & 8.22 & \\
\hline $\begin{array}{l}\text { Thevetia } \\
\text { peruvian } \\
a\end{array}$ & $\begin{array}{l}\text { fruit } \\
\text { wall }\end{array}$ & $\begin{array}{l}\text { Anomote } \\
\text { tracytic }\end{array}$ & $\begin{array}{l}1 \\
\text { or } \\
2 \\
\end{array}$ & $\begin{array}{c}0.9 \\
6\end{array}$ & $\begin{array}{c}0.0 \\
6\end{array}$ & $\begin{array}{l}\text { rectangul } \\
\text { ar }\end{array}$ & 175 & $\begin{array}{l}112 \\
.18\end{array}$ & 7.19 & 0.83 \\
\hline $\begin{array}{l}\text { Theveti } \\
a \\
\text { neriifol } \\
\text { ia }\end{array}$ & $\begin{array}{l}\text { Upp } \\
\text { er } \\
\text { layer } \\
\text { of } \\
\text { leaf }\end{array}$ & $\begin{array}{l}\text { Anomoc } \\
\text { ytic, } \\
\text { brachypa } \\
\text { racytic, } \\
\text { anisocyti } \\
\text { c, } \\
\text { anomotet } \\
\text { racytic }\end{array}$ & $\begin{array}{l}1 \\
\text { or } \\
\text { rar } \\
\text { e }\end{array}$ & .32 & .02 & $\begin{array}{l}\text { Polygona } \\
\text { l, } \\
\text { hexagon } \\
\text { al }\end{array}$ & 100 & $\begin{array}{l}64 . \\
10\end{array}$ & 4.11 & 0.48 \\
\hline Theveti & Low & Anomoc & 5 & 3.2 & .21 & $\begin{array}{l}\text { Polygona } \\
\end{array}$ & 110 & 70. & 4.52 & 4.44 \\
\hline
\end{tabular}


Taxonomic Studies Of Two Common Poisonous Plants

\begin{tabular}{|c|c|c|c|c|c|c|c|c|c|c|}
\hline $\begin{array}{l}a \\
\text { neriifol } \\
\text { ia }\end{array}$ & $\begin{array}{l}\text { er } \\
\text { layer } \\
\text { of } \\
\text { leaf }\end{array}$ & $\begin{array}{l}\text { ytic, } \\
\text { brachypa } \\
\text { racytic, } \\
\text { anisocyti } \\
\text { c, } \\
\text { anomotet } \\
\text { racytic }\end{array}$ & & 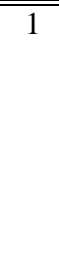 & & $\begin{array}{l}\text { l, } \\
\text { hexagon } \\
\text { al }\end{array}$ & & 51 & & \\
\hline $\begin{array}{l}\text { Theveti } \\
\text { a } \\
\text { neriifol } \\
\text { ia }\end{array}$ & Stem & $\begin{array}{l}\text { Anomoc } \\
\text { ytic }\end{array}$ & $\begin{array}{l}1 \\
\text { or } \\
\text { rar } \\
\text { e }\end{array}$ & .32 & .02 & $\begin{array}{l}\text { rectangul } \\
\text { ar }\end{array}$ & 48 & $\begin{array}{c}30 . \\
77\end{array}$ & 1.97 & 1.01 \\
\hline $\begin{array}{l}\text { Theveti } \\
\text { a } \\
\text { neriifol } \\
\text { ia }\end{array}$ & $\begin{array}{l}\text { Pedi } \\
\text { cel }\end{array}$ & $\begin{array}{l}\text { Anomoc } \\
\text { ytic }\end{array}$ & 1 & .64 & .04 & $\begin{array}{l}\text { rectangul } \\
\text { ar }\end{array}$ & 348 & $\begin{array}{c}223 \\
.08\end{array}$ & $\begin{array}{c}14.3 \\
0\end{array}$ & 0.28 \\
\hline $\begin{array}{l}\text { Theveti } \\
\text { a } \\
\text { neriifol } \\
\text { ia }\end{array}$ & $\begin{array}{l}\text { Caly } \\
\mathrm{x}\end{array}$ & $\begin{array}{l}\text { Brachyp } \\
\text { aracytic, } \\
\text { anomocy } \\
\text { tic }\end{array}$ & 5 & $\begin{array}{c}3.2 \\
1\end{array}$ & .21 & $\begin{array}{l}\text { rectangul } \\
\text { ar }\end{array}$ & 256 & $\begin{array}{l}164 \\
.10\end{array}$ & $\begin{array}{c}10.5 \\
2\end{array}$ & 1.96 \\
\hline $\begin{array}{l}\text { Theveti } \\
\text { a } \\
\text { neriifol } \\
\text { ia }\end{array}$ & $\begin{array}{l}\text { Coro } \\
\text { lla }\end{array}$ & $\begin{array}{l}\text { anomocy } \\
\text { tic }\end{array}$ & \begin{tabular}{|l|}
1 \\
\end{tabular} & $\begin{array}{c}0.6 \\
4\end{array}$ & $\begin{array}{c}0.0 \\
4\end{array}$ & $\begin{array}{l}\text { Hexagon } \\
\text { al, } \\
\text { pentagon } \\
\text { al }\end{array}$ & 93 & $\begin{array}{l}59 . \\
62\end{array}$ & 3.82 & 1.04 \\
\hline $\begin{array}{l}\text { Theveti } \\
\text { a } \\
\text { neriifol } \\
\text { ia }\end{array}$ & $\begin{array}{l}\text { Gyn } \\
\text { ocei } \\
\text { um }\end{array}$ & & & & & oval & 228 & $\begin{array}{l}146 \\
.15\end{array}$ & 9.37 & \\
\hline $\begin{array}{l}\text { Theveti } \\
\text { a } \\
\text { neriifol } \\
\text { ia }\end{array}$ & $\begin{array}{l}\text { Andr } \\
\text { ocei } \\
\text { um }\end{array}$ & & & & & oval & 169 & $\begin{array}{l}108 \\
.33\end{array}$ & 6.94 & \\
\hline $\begin{array}{l}\text { Theveti } \\
\text { a } \\
\text { neriifol } \\
\text { ia }\end{array}$ & $\begin{array}{l}\text { fruit } \\
\text { wall }\end{array}$ & $\begin{array}{l}\text { Anomoc } \\
\text { ytic, } \\
\text { brachypa } \\
\text { racytic, } \\
\text { anisocyti } \\
\text { c, } \\
\text { anomotet } \\
\text { racytic }\end{array}$ & \begin{tabular}{|l|}
1 \\
or \\
rar \\
$\mathrm{e}$
\end{tabular} & .32 & .02 & $\begin{array}{l}\text { polygona } \\
1\end{array}$ & 198 & $\begin{array}{l}126 \\
.92\end{array}$ & 8.14 & 0.25 \\
\hline
\end{tabular}

\subsection{Chart Of Stomata Index}

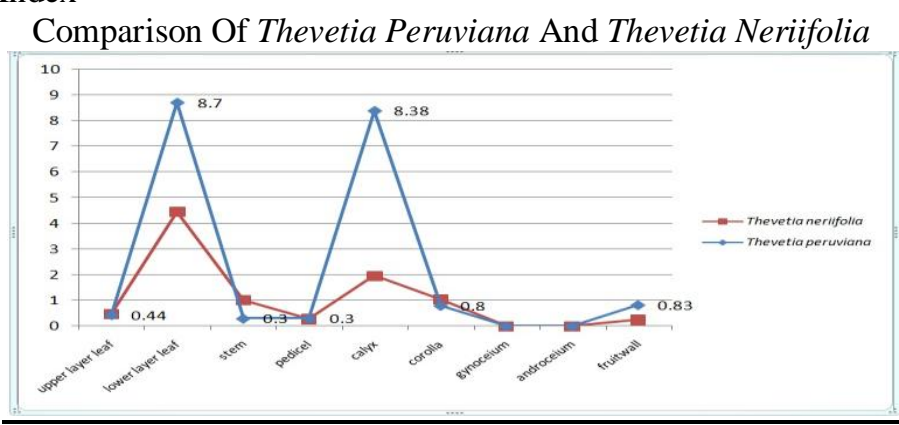

4.3. There are presented number of trichomes of studied plants in table 1

\begin{tabular}{|l|l|l|l|l|l|l|l|l|l|l|}
\hline $\begin{array}{l}\text { Name } \\
\text { of } \\
\text { plant }\end{array}$ & $\begin{array}{l}\text { Name } \\
\text { of } \\
\text { plant } \\
\text { parts }\end{array}$ & $\begin{array}{l}\text { type of } \\
\text { trichom } \\
\mathrm{e}\end{array}$ & $\begin{array}{l}\text { Nu } \\
\text { mbe } \\
\text { r of } \\
\text { trich } \\
\text { ome }\end{array}$ & $\begin{array}{l}\text { freque } \\
\text { ncy of } \\
\text { tricho } \\
\text { me }\end{array}$ & $\begin{array}{l}\text { den } \\
\text { sity } \\
\text { of } \\
\text { tric } \\
\text { ho } \\
\text { me }\end{array}$ & $\begin{array}{l}\text { Type of } \\
\text { epidermal } \\
\text { cell }\end{array}$ & $\begin{array}{l}\text { Numb } \\
\text { er of } \\
\text { epider } \\
\text { mal } \\
\text { cell }\end{array}$ & $\begin{array}{l}\text { Freque } \\
\text { ncy of } \\
\text { epider } \\
\text { mal } \\
\text { cell }\end{array}$ & $\begin{array}{l}\text { Densit } \\
\text { of } \\
\text { epider } \\
\text { mal } \\
\text { cell }\end{array}$ & $\begin{array}{l}\text { Index } \\
\text { of } \\
\text { trich } \\
\text { ome }\end{array}$ \\
\hline $\begin{array}{l}\text { Theve } \\
\text { tia } \\
\text { peruvi } \\
\text { ana }\end{array}$ & $\begin{array}{l}\text { Upper } \\
\text { layer } \\
\text { of leaf }\end{array}$ & & & & $\begin{array}{l}\text { Rectangul } \\
\text { ar, } \\
\text { pentagon } \\
\text { al, } \\
\text { hexagona } \\
1\end{array}$ & 110 & 70.51 & 4.52 & \\
\hline
\end{tabular}


Taxonomic Studies Of Two Common Poisonous Plants

\begin{tabular}{|c|c|c|c|c|c|c|c|c|c|c|}
\hline $\begin{array}{l}\text { Thevetia } \\
\text { peruvian } \\
a\end{array}$ & $\begin{array}{l}\text { Lowe } \\
\mathrm{r} \text { of } \\
\text { leaf }\end{array}$ & & & & & “ & 120 & 76.92 & 4.93 & \\
\hline $\begin{array}{l}\text { Thevetia } \\
\text { peruvian } \\
a\end{array}$ & Stem & $\begin{array}{l}\text { Multicel } \\
\text { led } \\
\text { flagellat } \\
\text { e } \\
\text { glandula } \\
\text { r hair }\end{array}$ & $\begin{array}{l}1 \text { or } \\
\text { rare }\end{array}$ & 0.32 & $\begin{array}{l}0.0 \\
2\end{array}$ & $\begin{array}{l}\text { rectangul } \\
\text { ar }\end{array}$ & 160 & 102.56 & 6.57 & 0.30 \\
\hline $\begin{array}{l}\text { Thevetia } \\
\text { peruvian } \\
a\end{array}$ & $\begin{array}{l}\text { Pedic } \\
\text { el }\end{array}$ & $\begin{array}{l}\text { Multicel } \\
\text { led } \\
\text { flagellat } \\
\text { e } \\
\text { glandula } \\
\text { r hair } \\
\end{array}$ & $\begin{array}{l}1 \text { or } \\
\text { rare }\end{array}$ & 0.32 & $\begin{array}{l}0.0 \\
2\end{array}$ & " & 160 & 102.56 & 6.57 & 0.30 \\
\hline $\begin{array}{l}\text { Thevetia } \\
\text { peruvian } \\
a\end{array}$ & Calyx & $\begin{array}{l}\text { Papillat } \\
\text { e } \\
\text { glandula } \\
\mathrm{r} \text { hair }\end{array}$ & $\begin{array}{l}1 \text { or } \\
\text { rare }\end{array}$ & 0.32 & $\begin{array}{l}0.0 \\
2\end{array}$ & $\begin{array}{l}\text { Rectangul } \\
\text { ar, } \\
\text { pentagon } \\
\text { al, } \\
\text { hexagona } \\
\text { 1, } \\
\text { polygonal }\end{array}$ & 125 & 80.13 & 5.14 & 0.30 \\
\hline $\begin{array}{l}\text { Thevetia } \\
\text { peruvian } \\
a\end{array}$ & $\begin{array}{l}\text { Coroll } \\
\mathrm{a}\end{array}$ & $\begin{array}{l}\text { Multicel } \\
\text { led } \\
\text { flagellat } \\
\text { e } \\
\text { glandula } \\
\text { r hair }\end{array}$ & 1 & 0.64 & $\begin{array}{l}0.0 \\
4\end{array}$ & $\begin{array}{l}\text { Rectangul } \\
\text { ar, } \\
\text { hexagona } \\
1\end{array}$ & 120 & 76.92 & 4.93 & 0.80 \\
\hline $\begin{array}{l}\text { Thevetia } \\
\text { peruvian } \\
a\end{array}$ & $\begin{array}{l}\text { Gyno } \\
\text { ceium }\end{array}$ & $\begin{array}{l}\text { Multicel } \\
\text { led } \\
\text { flagellat } \\
\mathrm{e} \\
\text { glandula } \\
\mathrm{r} \text { hair, } \\
\text { Papillat } \\
\mathrm{e} \\
\text { glandula } \\
\mathrm{r} \text { hair, } \\
\text { unicelle } \\
\mathrm{d} \\
\text { flagellat } \\
\mathrm{e} \\
\text { glandula } \\
\text { r hair } \\
\end{array}$ & 1 & 0.64 & $\begin{array}{l}0.0 \\
4\end{array}$ & oval & 325 & 208.33 & 13.35 & 0.30 \\
\hline $\begin{array}{l}\text { Thevetia } \\
\text { peruvian } \\
a\end{array}$ & $\begin{array}{l}\text { Andro } \\
\text { ceium }\end{array}$ & $\begin{array}{l}\text { Papillat } \\
\mathrm{e} \\
\text { glandula } \\
\mathrm{r} \text { hair, } \\
\text { unicelle } \\
\mathrm{d} \\
\text { flagellat } \\
\mathrm{e} \\
\text { glandula } \\
\text { r hair } \\
\end{array}$ & 1 & 0.64 & $\begin{array}{l}0.0 \\
4\end{array}$ & $\begin{array}{l}\text { Oval and } \\
\text { rectangul } \\
\text { ar }\end{array}$ & 200 & 128.21 & 8.22 & 0.48 \\
\hline $\begin{array}{l}\text { Thevetia } \\
\text { peruvian } \\
a\end{array}$ & $\begin{array}{l}\text { fruit } \\
\text { wall }\end{array}$ & $\begin{array}{l}\text { Multicel } \\
\text { led } \\
\text { flagellat } \\
\text { e } \\
\text { glandula } \\
\text { r hair, } \\
\text { Papillat } \\
\text { e } \\
\text { glandula } \\
\text { r hair } \\
\end{array}$ & 1 & 0.64 & $\begin{array}{l}0.0 \\
4\end{array}$ & $\begin{array}{l}\text { rectangul } \\
\text { ar }\end{array}$ & 175 & 112.18 & 7.19 & 0.55 \\
\hline $\begin{array}{l}\text { Theve } \\
\text { tia } \\
\text { neriif } \\
\text { olia }\end{array}$ & $\begin{array}{l}\text { Upper } \\
\text { layer } \\
\text { of leaf }\end{array}$ & $\begin{array}{l}\text { Two } \\
\text { celled } \\
\text { flagellat } \\
\text { e } \\
\text { glandula } \\
\text { r hair } \\
\end{array}$ & $\begin{array}{l}1 \text { or } \\
\text { rare }\end{array}$ & .32 & .02 & $\begin{array}{l}\text { Polygonal } \\
\text { hexagona } \\
1\end{array}$ & 100 & 64.10 & 4.11 & 0.48 \\
\hline $\begin{array}{l}\text { Thevetia } \\
\text { neriifoli } \\
a\end{array}$ & $\begin{array}{l}\text { Lowe } \\
r \quad \text { of } \\
\text { leaf }\end{array}$ & $\begin{array}{l}\text { Two } \\
\text { celled } \\
\text { flagellat } \\
\mathrm{e} \\
\text { glandula } \\
\end{array}$ & $\begin{array}{l}1 \text { or } \\
\text { rare }\end{array}$ & .32 & .02 & $\begin{array}{l}\text { Polygonal } \\
\text { hexagona } \\
1\end{array}$ & 110 & 70.51 & 4.52 & 0.44 \\
\hline
\end{tabular}


Taxonomic Studies Of Two Common Poisonous Plants

\begin{tabular}{|c|c|c|c|c|c|c|c|c|c|c|}
\hline & & $\begin{array}{l}\mathrm{r} \text { hair } \\
\end{array}$ & & & & & & & & \\
\hline $\begin{array}{l}\text { Thevetia } \\
\text { neriifoli } \\
a\end{array}$ & Stem & $\begin{array}{l}\text { Two } \\
\text { celled } \\
\text { flagellat } \\
\mathrm{e} \\
\text { glandula } \\
\mathrm{r} \text { hair, } \\
\text { unicelle } \\
\mathrm{d} \\
\text { flagellat } \\
\mathrm{e} \\
\text { glandula } \\
\text { r hair } \\
\end{array}$ & $\begin{array}{l}10,1 \\
\text { or } \\
\text { rare }\end{array}$ & 2.37 & $\begin{array}{l}0.1 \\
5\end{array}$ & $\begin{array}{l}\text { rectangul } \\
\text { ar }\end{array}$ & 48 & 30.77 & 1.97 & 7.08 \\
\hline $\begin{array}{l}\text { Thevetia } \\
\text { neriifoli } \\
a\end{array}$ & $\begin{array}{l}\text { Pedic } \\
\text { el }\end{array}$ & $\begin{array}{l}\text { Two } \\
\text { celled } \\
\text { flagellat } \\
\mathrm{e} \\
\text { glandula } \\
\mathrm{r} \text { hair } \\
\text { unicelle } \\
\mathrm{d} \\
\text { flagellat } \\
\mathrm{e} \\
\text { glandula } \\
\mathrm{r} \text { hair } \\
\end{array}$ & $\begin{array}{l}1 \text { or } \\
\text { rare }\end{array}$ & .32 & .02 & $\begin{array}{l}\text { rectangul } \\
\text { ar }\end{array}$ & 348 & 223.08 & 14.30 & 0.14 \\
\hline $\begin{array}{l}\text { Thevetia } \\
\text { neriifoli } \\
a\end{array}$ & Calyx & $\begin{array}{l}\text { unicelle } \\
\mathrm{d} \\
\text { flagellat } \\
\mathrm{e} \\
\text { glandula } \\
\mathrm{r} \text { hair }\end{array}$ & $\begin{array}{l}1 \text { or } \\
\text { rare }\end{array}$ & .32 & .02 & $\begin{array}{l}\text { rectangul } \\
\text { ar }\end{array}$ & 256 & 164.10 & 10.52 & 0.19 \\
\hline $\begin{array}{l}\text { Thevetia } \\
\text { neriifoli } \\
a\end{array}$ & $\begin{array}{l}\text { Coroll } \\
\text { a }\end{array}$ & $\begin{array}{l}\text { Two } \\
\text { celled } \\
\text { flagellat } \\
\text { e } \\
\text { glandula } \\
\text { r hair }\end{array}$ & 1 & .64 & .04 & $\begin{array}{l}\text { Hexagona } \\
1, \\
\text { pentagon } \\
\text { al }\end{array}$ & 93 & 59.62 & 3.82 & 1.04 \\
\hline $\begin{array}{l}\text { Thevetia } \\
\text { neriifoli } \\
a\end{array}$ & $\begin{array}{l}\text { Gyno } \\
\text { ceium }\end{array}$ & $\begin{array}{l}\text { Uniseria } \\
\text { te } \\
\text { flagellat } \\
\mathrm{e} \\
\text { glandula } \\
\mathrm{r} \text { hair, } \\
\text { unicelle } \\
\mathrm{d} \\
\text { papilllat } \\
\mathrm{e} \\
\text { glandula } \\
\mathrm{r} \text { hair }\end{array}$ & 1 & .64 & .04 & oval & 228 & 146.15 & 9.37 & 0.43 \\
\hline $\begin{array}{l}\text { Thevetia } \\
\text { neriifoli } \\
a\end{array}$ & $\begin{array}{l}\text { Andro } \\
\text { ceium }\end{array}$ & $\begin{array}{l}\text { Multicel } \\
\text { led } \\
\text { branche } \\
\mathrm{d} \\
\text { glandula } \\
\mathrm{r} \text { hair, } \\
\text { unicelle } \\
\mathrm{d} \\
\text { flagellat } \\
\mathrm{e} \\
\text { glandula } \\
\mathrm{r} \text { hair }\end{array}$ & 2,6 & 2.56 & $\begin{array}{l}0.1 \\
6\end{array}$ & oval & 169 & 108.33 & 6.94 & 0.02 \\
\hline $\begin{array}{l}\text { Thevetia } \\
\text { neriifoli } \\
a\end{array}$ & $\begin{array}{l}\text { fruit } \\
\text { wall }\end{array}$ & $\begin{array}{l}\text { Two } \\
\text { celled } \\
\text { flagellat } \\
\mathrm{e} \\
\text { glandula } \\
\mathrm{r} \quad \text { hair, } \\
\text { Two } \\
\text { celled } \\
\text { flagellat } \\
\mathrm{e} \\
\text { glandula } \\
\mathrm{r} \text { hair }\end{array}$ & $\begin{array}{l}1 \text { or } \\
\text { rare }\end{array}$ & 0.32 & $\begin{array}{l}0.0 \\
2\end{array}$ & polygonal & 198 & 126.92 & 8.14 & 0.00 \\
\hline
\end{tabular}


Comparison Of Thevetia Peruviana And Thevetia Neriifolia

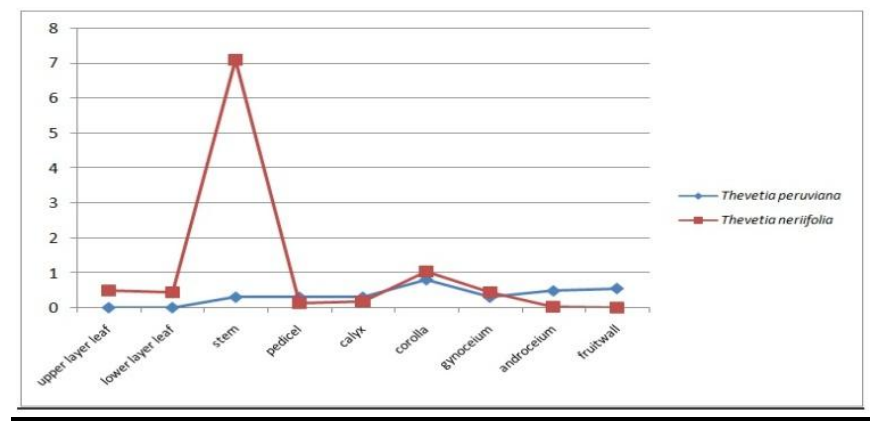

4.5.1. Trichome Plate A

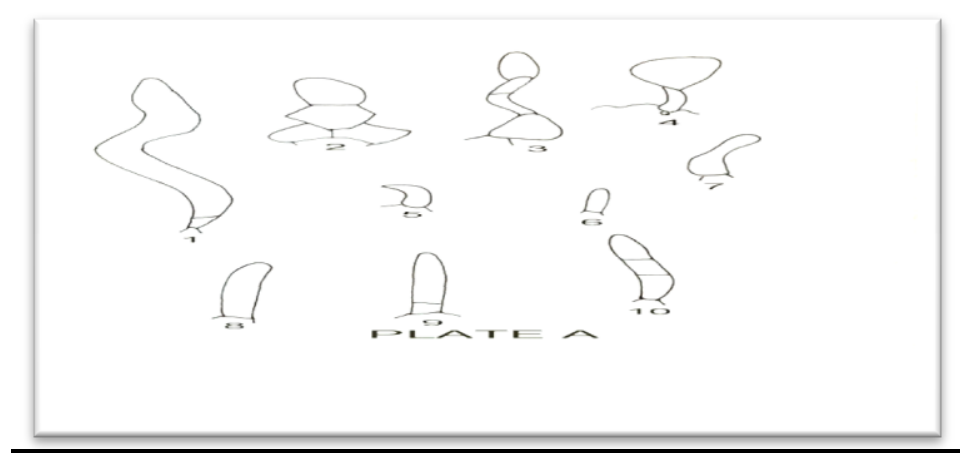

4.5.1.1. In thevetia peruviana trichome plate A

Fig1, 3, 4, 9, 10: multicelled flagellate glandular hair

Fig. 1: $40 \times 62.50$

Fig.3: 40x 38.75

Fig.4: $40 \times 31.25$

Fig.9: $40 \times 31.25$

Fig.10: 40x26.25

Fig5, 8, 6: unicelled flagellate glandular hair

Fig. 5 : $40 \times 8.75$

Fig.8: $40 \times 25$

Fig.6:40x8.75

Fig. 2, 7: papillate glandular hair

Fig. 2: 40x10

Fig.7: 40x25

\subsubsection{Trichome plate B}

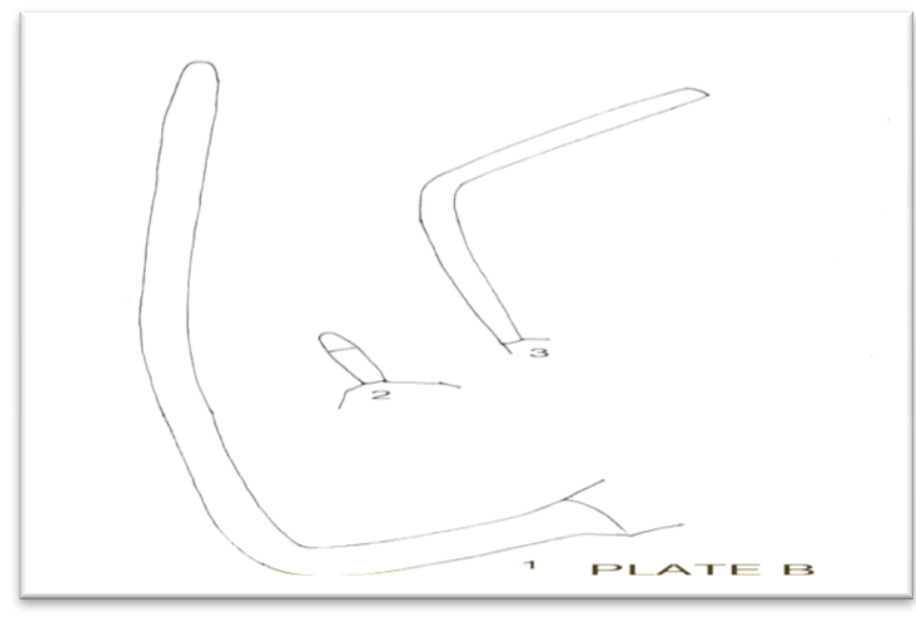


4.5.2.1. In thevetia neriifolia trichome plate B

Fig.1, 3: unicelled flagellate glandular hair

Fig.1: 40x500

Fig.3: 40x100

Fig.2: two celled flagellate glandular hair 40x 18.75

4.5.3. Trichome plate $\mathrm{C}$

4.5.3.1. In thevetia neriifolia trichome plate $\mathrm{C}$

Fig.1: multicelled branched glandular hair 40x31.25

Fig.2: two celled cylindrical glandular hair 40x47.50

Fig.3, 4, 6, 7: unicelled papillate glandular hair

Fig.3: $40 \times 27.50$

Fig.4: $40 \times 13.75$

Fig.6: $40 \times 22.50$

Fig.7: $40 \times 12.50$

Fig.5, 8, 10: unicelled flagellate glandular hair

Fig.5: $40 \times 62.50$

Fig. 8 : $40 \times 40$

Fig. 10: $40 \times 250$

Fig.9: uniseriate flagellate glandular hair 40x43.75

\subsubsection{Stomata Plate A}

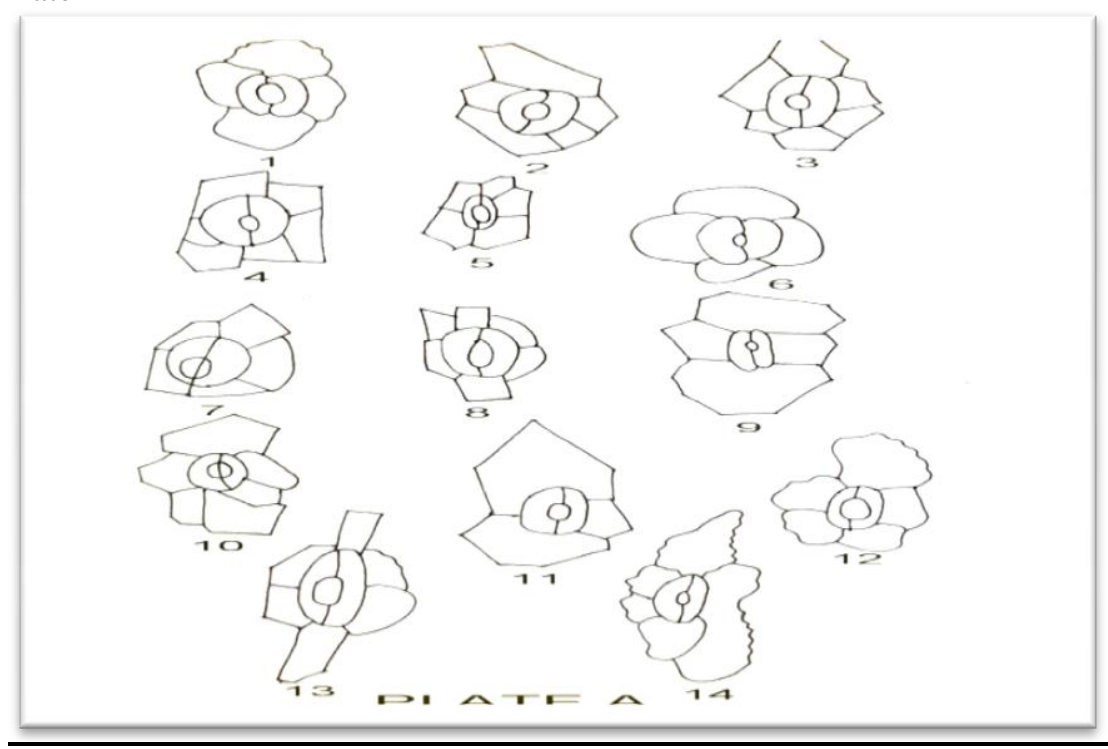

4.5.4.1. In Thevetia Peruviana Stomata Plate A

Fig.1: Anomotetracytic stomata, in leaf

Size: in $40 X$

Stomata: $1=11.25, b=7.50$

Stomata with subsidiary cell: $-1=23.75, b=16.25$

Fig.2, 5: Brachyparacytic stomata

Fig.2: in leaf, fruitwall

Size: in $40 \mathrm{X}$

Stomata: $\mathrm{l}=11.25, \mathrm{~b}=7.50$

Stomata with cell: $-\mathrm{l}=21.25, \mathrm{~b}=18.75$

Fig.5: in petal

Size: in 40X

Stomata: $1=7.50, b=5$

Stomata with subsidiary cell: $l=18.75, b=12.50$

Fig.3, 4: Anomocytic stomata

Fig.3: in sepal

Size: in $40 \mathrm{X}$

Stomata: $l=13.75, b=8.75$

Stomata with cell: $1=31.25, b=16.25$

Fig.4: in stem 
Size: in 40X

Stomata: $1=13.75, b=10$

Stomata with cell: $\mathrm{l}=25, \mathrm{~b}=16$.

4.5.4.2. In thevetia neriifolia stomata plate A

Fig.7, 12: Brachyparacytic stomata

Fig.7: in sepal, fruitwall

Size: in 40X

Stomata: $1=15, b=10$

Stomata with cell: $\mathrm{l}=21.25, \mathrm{~b}=18.75$

Fig.12: in leaf

Size: in 40X

Stomata: $1=10, b=6.25$

Stomata with cell: $1=26.25, b=17.50$

FIG.8, 10, 13, 14: Anomocytic stomata

Fig.8: in sepal

Size: in $40 \mathrm{X}$

Stomata: $1=15, b=10$

Stomata with cell: $\mathrm{l}=21.25, \mathrm{~b}=18.75$

Fig.10: in leaf

Size: in 40X

Stomata: $-\mathrm{l}=10, \mathrm{~b}=6.25$

Stomata with cell: $1=25, b=18.75$

Fig.13: in pedicel

Size: in 40X

Stomata: $1=18.75, b=10$

Stomata with cell: $1=46.25, b=18.75$

Fig.14: in petal

Size: in 40X

Stomata: $1=10, b=6.25$

Stomata with cell: $l=25, b=18.75$

Fig.9: Anomotetracytic stomata, in leaf

Fig.9: in leaf

Size: in 40X

Stomata: $1=10, b=5$

Stomata with cell: $\mathrm{l}=31.25, \mathrm{~b}=21.25$

Fig.11: Anisocytic stomata, in leaf

Fig.11: in leaf

Size: in $40 \mathrm{X}$

Stomata: $1=11.25, b=6.25$

Stomata with cell: $\mathrm{l}=28.75, \mathrm{~b}=20$

\section{Results and discussion}

In apocynaceae family Thevetia peruviana and Thevetia neriifolia are distributed in tropical area. They are shrub and tree in habit. They have been found many constituents like therein A and B, thevetoxin, peruvoside, ruvoside, nerifolin. They are useful for treatment of many diseases like cardiac insufficiency and febrifuges menstrual cycle. They are toxic as digitalis poisoning.

There have been seen anomocytic, anomotetracytic, brachyparacytic and anisocytic stomata and unicelled flagellate glandular hair, two celled flagellate hair, multicelled branched glandular hair, two celled cylindrical glandular hair, unicelled papillate glandular hair, multicelled flagellate glandular hai, papillate glandular hair in Thevetia peruviana and Thevetia neriifolia with the help of microscope.

The largest stomata 11 or 12 have been found in calyx and lower layer of leaf of Thevetia peruviana. The largest stomata frequency 7.37 has been found in calyx and lower layer of leaf of Thevetia peruviana. The largest stomata density .47 has been found in calyx and lower layer of leaf of Thevetia peruviana. The largest stomata index 8.7 has been found in calyx and lower layer of leaf of Thevetia peruviana. Stomata, stomata frequency, stomata density, stomata index has been absent in gynoceium, androceium of Thevetia peruviana and Thevetia neriifolia. The largest trichome number 10 has been found in stem of Thevetia neriifolia. The largest trichome frequency 2.37 has been found in stem of Thevetia neriifolia. The largest trichome density 0.15 has been found in stem of Thevetia neriifolia. The largest trichome index 7.08 has been found in stem of Thevetia 
neriifolia. There have been absent trichome, trichome index, and trichome frequency, trichome density in upper and lower layer of leaf of Thevetia peruviana.

\section{Conclusion}

Thevetia peruviana and Thevetia neriifolia have fragnent flowers which exist in the form of shrub or trees. Its fruits are hard capsules. It can be found in tropical region and shows digitalis poisoning. The present study therefore attempts to use microscopic examinations of epidermal cells and stomata with a view to their elucidating diagnostic significance and can be singled out as being the most significant in relation to the taxonomic separation of the taxa.

\section{Acknowledgement}

"One touch of nature makes the whole work kin"

Apart from our hard work, devotion and grace of our parents the completion of this research required a helping hand of number of person, here is acknowledge in the honor of those super persons.

First of all our sincere thanks to respected Dr. Ela Tiwari without whose kind permission the project would have not even started, also the lecturer of Botany department who guided us all the way through this research

We should be failing duly if we do not express out deep sense of gratitude to all our friends who have shared their valuable time and helped us directly or indirectly in the preparation of our research.

At this end, last but not least we would like to thanks to other staff members of this department.

\section{Journal papers:}

\section{References}

[1] Faust, W. Z. And Jr. S. B. Johnes. 1973. The systematic value of trichome complements in North American group of Vernonia (Compositae). Rhodora, 75:517-528.

a. Ramayya, N.1962b. Studies on the trichome of some Composite I General structure. Bull. Bot. Serv. India, 1:-4:187-192.

b. Sahu, T.R.1982. Studies on the trichomes in Helianthoideae (Asteraceae). J.Econ.Tax.Bot.Vol.3:517-521.

Sahu, T.R.1983. Trichome studies in senecio linn: structure, distribution and Taxonomic significance. Indian Bot. Soc. 62:84-89.

c. Sahu, T.R.1984.Taxonomic implications of trichome complements to Vernonia (Compositae) in India. Feddes Repertorium, 95(4):237-249.

d. Sahu, T.R.1985. Studies on the trichomes in Asteroideae (Asteraceae). Proc.Nat.Acad.Sci.India, 55(B), I: 39-44

e. Tiwari, E. And T. R. Sahu, 1982. Taxonomic implication of trichome morphology in pluchea Cass. (Compositae). Life Science Advance, I (3):315-317.

f. Inamdar, J.A. and M.Gangadhara, 1976. Structure, ontogeny, classification and taxonomic significance of stomata in Cucurbitaceae. Feddes Repertorium, 87(5):293-310.

g. Boubos, S. T. And A.B. Beakbane.1971. A chemical method for separating leaf epidermis from mesophyll tissue. U.A.R.J.Bot., 17:317-322.

h. Salisbury, E.J.1927. On the causes and ecological significance of stomatal frequency, with special reference to the woodland flora. Philos. Trans. R. Soc. London. B: 216, 1-65.

\section{Books:}

[2] Smith, A.B.1923. Poisonous plants of all countries $2^{\text {nd }}$ eds (Tin dall and Company, London).

a. Caius, J.F. 2003. The medicinal and poisonous plants of India (Scientific publishers Journals Dept., India ed 5 th pp.528).

b. Metcalfe, C. R. And L. Chalk. 1979. Anatomy of the dicotyledons $2^{\text {nd }}$ eds (Oxford. Clarendon press).

c. Champion, H.G. and S. K. Seth 1968. A revised survey of the forest types of India (Delhi XXVII, 404 P).

d. Metcalfe, C. R. And L. Chalk. 1950. Anatomy of the dicotyledons, vol. I (Clarendon press).

\section{Proceeding papers:}

[3] Abid, R., Sharmeen, S. And Perveen, A.2007. Stomatal types of monocots within flora of Karachi, Pakistan.Pak.J.Bot.39(I): 1527.

a. Ahmad, K., Khan, M.A., Ahmad, M., Zafar, M., Arshad, M., and Ahmad, F.2009. Taxonomic diversity of stomata in dicot flora of a district tank (N.W.F.P.) in Pakistan. African journal of Biotechnology vol. 8(6); 1052-1055.

b. Perveen, A., Abid, R., and Fatima, R.2007.Stomatal types of some dicots within flora of Karachi, Pakistan. Pak.J.Bot.39(4):10171023. 